\title{
STRATIGRAPHIC DATA AND ESTIMATES OF PHYLOGENIES: CONTRASTING THE EFFICIENCY OF DIFFERENT METHODS USING SIMULATIONS.
}

\author{
HUELSENBECK*, John P.. Department of Integrative Biology, University of \\ California, Berkeley, CA 94720-3140. WAGNER, Peter J. Department of \\ Paleobiology, Smithsonian Institution, Washington, D.C. 20560.
}

The role of stratigraphic data in estimating phylogenetic relationships has been controversial. Simulations offer a means of testing the relevance of stratigraphy in phylogenetic analysis and also a method to examine the relative performance of various phylogenetic methods that incorporate stratigraphic information. In this study, we examined the performance of several different phylogenetic methods, including parsimony and several parimony variants which used stratigraphic information. The simulations assumed a birth-death process to generate the topology and branch lengths of the model trees. Binary characters were then evolved on the trees using the Cavender-Farris model of change. The parameters that were varied in these simulations included: the speciation rate, the extinction rate, the rate of change of the characters, the density of taxon sampling, and the number of fossil horizons. Hence, we could investigate the statistical properties of phylogenetic methods under a wide range of simulated conditions. Many of the simulations included parameter conditions similar to those observed for many readily fossilized taxa.

For a wide range of conditions, phylogenetic methods that included stratigraphic information performed better than parsimony. Even when there is general agreement, stratigraphic methods often are more exact (e.g., finding ancestor-descendant relationships instead of sister-species relationships). However, for some methods (those that weight the length of trees by some function of the stratigraphic information), there are conditions for which the stratigraphic methods perform poorly compared to parsimony. These conditions include cases in which the taxon sampling is low and the stratigraphic record is incomplete: a situation that is expected to occur commonly for some real taxa. However, one stratigraphic weighting scheme seems relatively insensitive to the effects of incompleteness in the fossil record. This method picks that tree out of the set of equally parsimonious trees which best fits the stratigraphic record.

Although many workers have recommended that stratigraphic data not be considered when estimating phylogenies, these simulations show that even for conditions favorable to parsimony, estimates using stratigraphic data often are as accurate or more accurate than estimates ignoring stratigraphic information. This is especially true when sampling densities and rates of morphologic evolution similar to those of most marine invertebrates are used. When using cladograms to describe patterns of evolution, workers therefore should consider what information the stratigraphic record can contribute. 